\title{
28 Research Square \\ Readmission rate after epilepsy surgery: A systematic review and meta-analysis
}

\section{Soheila Mir}

Zabol university of medical sciences ,Zabol, Iran

Anis Jor

Zabol university of medical sciences ,Zabol, Iran

Morteza Salarzaei ( $\nabla$ mr.mortezasalar@gmail.com )

Zabol university of medical sciences ,Zabol, Iran

\section{Research article}

Keywords: Epilepsy, surgery, national readmission data-base

Posted Date: June 1st, 2020

DOI: https://doi.org/10.21203/rs.3.rs-31532/v1

License: (c) (i) This work is licensed under a Creative Commons Attribution 4.0 International License. Read Full License 


\section{Abstract}

Objective

Today, quality of care has become a central issue with regard to redesigning health care delivery. For improvement of quality of care, measurement and analysis of outcomes is essential. the aim of this study is to evaluate the 30 day readmission rate of epilepsy surgery.

\section{Material and Methods}

A systematic literature review was performed in April 2020. We reviewed MEDLINE/PubMed, Cochrane Library, and ClinicalTrials.gov for citation or ongoing trials from January 2010 to March 2020. The search criteria were limited to human studies published in English language. The Medical Subject Headings terms used for the search in PubMed were 'epilepsy surgery' ',readmission', 'reoperation ' and 'neurological surgery'. We used random-effects meta-analyses to estimate pooled risk ratios and $95 \%$ confidence intervals for early readmission

Results

In the 7trials reporting readmission rates, the overall prevalence of readmission and re-operation within 30 days was $10 \%$ and $9 \%$. The most common cause of re-admission was seizure(31.6\%). The overall mean Elixhauser index among readmitted patients after epilepsy surgery was 8.05.

\section{Conclusion}

Minimizing factors that contribute to readmission in various patient populations and procedures becomes important for patient care, resource utilization, and physician reimbursement.understanding about a readmission rate of $10 \%$ can be useful to health systems designing quality improvement efforts.Awareness of the reasons for readmission is important for patient counseling and surgical decision making.In epilepsy surgery, although our study did not show a significant difference but, readmissions might be reduced with optimization of pre-existing comorbidities

\section{Introduction:}

today, quality of care has become a central issue with regard to redesigning health care delivery. For improvement of quality of care, measurement and analysis of outcomes is essential. One outcome that is of interest in terms of measuring quality of care and hospital performance is the 30-day readmission rate (1). Jencks et al. recently reported a profile of readmitted patientsamong the Medicare population (2). In this study, nearly $20 \%$ of Medicare beneficiaries who had been discharged from a hospital were readmitted within 30 days, and the estimated cost to Medicare of unplanned readmissions in a single year was $\$ 17.4$ billion. Concerns that some readmissions might be preventable have influenced policy changes to simultaneously improve quality of care and lower costs (3). Hospitals at which Medicare risk-adjusted readmission rates are greater than the national average are at risk for losing reimbursements. Recent standards established by the Centers for Medicare \& Medicaid Services (CMS) have initiated programs to reduce the economic burden of 
hospital readmission by penalizing hospitals with higher than expected readmission rates (4). Readmissions contribute to higher operating costs for health- care systems as seen with unplanned 30-day readmissions costing Medicare \$17.4 billion in the year of 2004 (5). Epilepsy surgery is highly effective in appropriately seected candidates with drug-resistant epilepsy (6). A nationwide study examining 30-day readmissions after surgical resection for drug-resistant epilepsy noted an $11.5 \%$ read- mission rate. Other studies using the Agency for Healthcare Research Quality (AHRQ) State Inpatient Database (SID) reported readmission rates of up to $14 \%$ for seizures after resective epilepsy surgery (7). These policies addressing all-cause readmissions necessitate a better understanding of the factors that influence readmission. Although extensive literature about readmission attributed to particular conditions, especially congestive heart failure, is available, there remains a paucity of research on readmissions within neurosurgical practices (8). A recent, single-institution study measuring neurosurgical outcomes after more than 5000 procedures reported rates of major complications but did not include readmissions. The remaining information is limited to cohorts undergoing spine surgery, for whom reported 30-day readmission rates range from $4.2-9.1 \%$. In all studies that we found, the most common reason for readmission was infection (9-10). To elucidate the rate, reason, and predictors of readmissions after seizure interventions at a tertiary/quaternary neurosurgical service, Such analyses are critical for defining the problem so that the area to target for quality improvement within neurosurgery can be identified.the aim of this study is to evaluate the 30 day readmission rate of epilepsy surgery.

\section{Material And Methods: \\ Eligibility Criteria}

Eligible studies were randomized trials reported in English, since 2010, that assessed the rate of readmissions in 30 days after epilepsy surgery.The intervention had to focus its efforts on the hospital-to-home transition, permit patients across arms to have otherwise similar inpatient experiences, and be generalizable to contexts beyond a single patient diagnosis. Adult patients had to be admitted from the community to an inpatient ward for at least 24 hours with a medical or surgical cause. Studies including obstetric or psychiatric admissions or only including discharges to skilled nursing or rehabilitation facilities were excluded.

\section{Data Sources And Search Strategy}

A systematic literature review was performed in April 2020. We reviewed MEDLINE/PubMed, Cochrane Library, and ClinicalTrials.gov for citation or ongoing trials from January 2010 to March 2020. The search criteria were limited to human studies published in English language. The Medical Subject Headings terms used for the search in PubMed were 'epilepsy surgery', 'readmission', 'reoperation ' and 'neurological surgery' .

\section{Data Extraction}

Data extraction was conducted independently according to the Preferred Reporting Items for Systematic Reviews and Meta Analyses. Two authors selected studies following the previously described inclusion criteria. After this first selection, if needed, another author was consulted to achieve a shared decision. A 
predefined protocol for data extraction was used to retrieve data of each study, including first author name, year of publication, journal of publication, sample size, study design, demographic profile,readmission rate,reoperation rate, and significant variable at uni and multivariate analysis.

\section{Risk Of Bias}

Two raters (M.S and FP.) worked independently and in duplicate to determine the extent to which each trial was at risk of bias using a standardized form based on the Cochrane Colaboration's tool.The assessment considered the quality of the randomization sequence generation, allocation concealment, blinding of outcome assessors, the potential for missing outcomes (ie, likelihood of missing readmissions to other hospitals), and the proportion of patients lost to follow-up. For missing outcomes, "high risk of bias" was assigned when the readmissions data came from internal health system records only. To assess for publication bias, we examined a funnel plot for asymmetry and conducted asymmetry regression according to Sterne and Egger and determined the associated $P$ value.

\section{Data Synthesis}

We used random-effects meta-analyses to estimate pooled risk ratios and $95 \%$ confidence intervals for early readmission. We tested for heterogeneity of effect on this outcome using the Cochran $Q \times 2$ test and estimated between-trial inconsistency not due to chance using the 12 statistic.To explore the effects of patient, intervention, and out come characteristics on the impact of measured intervention effectiveness, we conducted planned subgroup analyses, testing variables 1 at a time. Patient characteristics tested were age, gender, and hospital ward (general medical or other). Ad hoc variables tested were year of publication and type of outcome reported (ie, unplanned readmissions).

\section{Results}

\section{Study Selection}

Our initial database search generated 327 reports (Fig. 1)Through abstract and title screening, 319 reports were identified for full-text review. During full-text screening 7 were selected for inclusion and 53 were set aside for author contact prior to making a decision.

\section{Study Characteristics}

Table 2 describes the included trials. Many were single- center trials taking place in academic medical centers, enrolling few patients and 30-day readmissions. Most interventions tested took place in both the inpatient and outpatient settings. Most studies were at low risk of bias. The most common methodological limitation of these trials was the lack of a reliable method for dealing with missing data. 
Table 1

Demographic characteristics for the included studies

\begin{tabular}{|c|c|c|c|c|c|c|c|c|}
\hline author & year & Country & age & $\mathbf{N}$ & Male & $\begin{array}{l}\mathrm{Re} \\
\text { admission }\end{array}$ & $\begin{array}{l}\mathrm{Re}- \\
\text { operation }\end{array}$ & $\begin{array}{l}\text { Most } \\
\text { common } \\
\text { reason for } \\
\text { re- } \\
\text { admission }\end{array}$ \\
\hline $\begin{array}{l}\text { Aditya } \\
\text { Vedantam }^{22}\end{array}$ & 2018 & USA & 10 & 208 & $51.8 \%$ & $7.1 \%$ & $6.8 \%$ & $\mathrm{~N} / \mathrm{A}$ \\
\hline $\begin{array}{l}\text { Churl-Su } \\
\text { Kwon } 23\end{array}$ & 2019 & USA & $\begin{array}{l}38.42 \\
\pm 1.87\end{array}$ & 2284 & $50.42 \%$ & $10.83 \%$ & & Seizure \\
\hline $\begin{array}{l}\text { Paul S. A. } \\
\text { Kalanithi } 24\end{array}$ & 2013 & $\begin{array}{l}\text { USA2005- } \\
2009\end{array}$ & 38 & 536 & & $4 \%$ & $3.9 \%$ & Seizure \\
\hline $\begin{array}{l}\text { Marissa } \\
\text { Vawter-Lee } 25\end{array}$ & 2018 & $\begin{array}{l}\text { 2014- } \\
\text { 2016USA }\end{array}$ & $\begin{array}{l}29.9 \\
\pm 1.4\end{array}$ & 1587 & $50.8 \%$ & $8 \%$ & & Seizure \\
\hline $\begin{array}{l}\text { Varsha } \\
\text { Subramaniam } \\
26\end{array}$ & 2019 & USA & $\mathrm{N} / \mathrm{A}$ & 2231 & & $12.3 \%$ & $12.4 \%$ & Seizure \\
\hline $\begin{array}{l}\text { Nuriel } \\
\text { moghavem }\end{array}$ & 2015 & USA & $\mathrm{N} / \mathrm{A}$ & 1214 & $53 \%$ & $13.9 \%$ & $\mathrm{~N} / \mathrm{A}$ & $\mathrm{N} / \mathrm{A}$ \\
\hline Rumalla 10 & 2017 & USA & $\mathrm{N} / \mathrm{A}$ & 1587 & $50.8 \%$ & $11.5 \%$ & $\mathrm{~N} / \mathrm{A}$ & seizure \\
\hline
\end{tabular}


Table 2

Meta-analysis of the Characteristics of included studies

\begin{tabular}{|c|c|c|c|c|c|c|}
\hline \multicolumn{2}{|c|}{ Characteristics of included studies } & \multicolumn{5}{|c|}{$95 \%$ conf. interval } \\
\hline & & Low & UP & ES & Weight & $p^{2}$ \\
\hline \multicolumn{2}{|l|}{ Re admission } & 0.093 & 0.105 & 0.099 & 100 & 93.5 \\
\hline \multicolumn{2}{|l|}{ Re-operation } & 0.078 & 0.098 & 0.088 & 100 & 96.7 \\
\hline \multirow[t]{3}{*}{ payer } & Private & 0.084 & 0.099 & 0.092 & 100 & 99.7 \\
\hline & Medicaid & 0.041 & 0.052 & 0.047 & 100 & 99.6 \\
\hline & Medicare & 0.073 & 0.086 & 0.080 & 100 & 99.7 \\
\hline \multirow[t]{2}{*}{ complications } & Surgical & 0.094 & 0.102 & 0.086 & 100 & 99.5 \\
\hline & Medical & 0.043 & 0.057 & 0.050 & 100 & 99.2 \\
\hline \multicolumn{2}{|c|}{ Most common reason for re-admission(seizure) } & 0.302 & 0.329 & 0.316 & 100 & 99.6 \\
\hline \multirow[t]{2}{*}{ Bed size } & Small or medium & 0.045 & 0.056 & 0.051 & 100 & 99.7 \\
\hline & Large & 0.336 & 0.354 & 0.345 & 100 & 98.4 \\
\hline \multirow[t]{4}{*}{ annual household income } & 0-25th percentile & 0.187 & 0.212 & 0.199 & 100 & 97.2 \\
\hline & 26th-50th percentile & 0.151 & 0.174 & 0.163 & 100 & 99.4 \\
\hline & 51st-75th percentile & 0.126 & 0.147 & 0.136 & 100 & 99.4 \\
\hline & 76th-100th percentile & 0.197 & 0.222 & 0.209 & 100 & 99.1 \\
\hline
\end{tabular}

\section{Meta-analysis}

The readmission and re-operation prevalence :

In the 7trials reporting readmission rates, the overall pooled prevalence of readmission within 30 days was $10 \%\left(95 \% \mathrm{Cl}, 9 \%, 11 \%, \mathrm{I}^{2}: 93.5 \%\right.$ ) (Fig. 2a). and the pooled prevalence of need for re-operation was $9 \%$ (95\% $\mathrm{Cl}, 8 \%, 10 \%, \mathrm{l}^{2}: 96.7 \%$ ) based on three articles reported this data(Fig. 2b)

Meta-analysis of prevalence of different types of payer in readmitted patients after epilepsy surgery:

The total prevalence of readmitted patients with Private payer, Medicaid and Medicare payers were $9.2 \%(95 \%$ $\mathrm{Cl}, 8.4 \%, 9.9 \%, \mathrm{I}^{2}: 99.7 \%$ ), 4.7\%(95\% Cl,4.1\%,5.2\%, $\left.\mathrm{l}^{2}: 99.6 \%\right), 8 \%\left(95 \% \mathrm{Cl}, 7.3 \%, 8.6 \%, \mathrm{l}^{2}: 99.7 \%\right)$, respectively. Meta-analysis Of Prevalence Of Different Sizes Of Hospital Beds:

The total prevalence of readmitted patients with large beds and small or medium beds were $34.5 \%(95 \%$ $\left.\mathrm{Cl}, 33.6 \%, 35.4 \%, \mathrm{I}^{2}: 100 \%\right)$, and $5.1 \%\left(95 \% \mathrm{Cl}, 4.5 \%, 5.6 \%, \mathrm{I}^{2}: 99.7 \%\right)$, respectively.

Meta-analysis of Median annual household income among readmitted patients after epilepsy surgery : 
The total prevalence of readmitted patients with being in the 0-25th percentile, 26th -50th percentile and 51st -75th percentile were 19.9\%(95\% Cl,18.7\%,21.2\%, $\left.1^{2}: 99.2 \%\right), 16.3 \%\left(95 \% \mathrm{Cl}, 15.1 \%, 17.4 \%, 1^{2}: 99.4 \%\right), 13.6 \%(95 \%$ $\left.\mathrm{Cl}, 7.12 .6 \%, 14.7 \%, \mathrm{I}^{2}: 99.4 \%\right)$, respectively.

Meta-analysis of prevalence of surgical and medical complications in readmitted patients after epilepsy surgery:

The total prevalence of surgical and medical complications among readmitted patients after epilepsy surgery were $9.4 \%\left(95 \% \mathrm{Cl}, 8.6 \%, 10.2 \%, \mathrm{I}^{2}: 99.5 \%\right)$, and $5 \%(95 \% \mathrm{Cl}, 4.3 \%, 5.7 \%)$, respectively.

Meta-analysis of the mean length of stay (LOS) among readmitted patients after epilepsy surgery:

The overall mean length of stay among readmitted patients after epilepsy surgery was 2.95 days.

Meta-analysis of the mean Elixhauser index among readmitted patients after epilepsy surgery:

The overall mean Elixhauser index among readmitted patients after epilepsy surgery was 8.05.

Meta-analysis of the prevalence of seizure as the most common cause of readmission among patients after epilepsy surgery:

The overall prevalence of seizure among readmitted patients after epilepsy surgery was 31.6\%(95\% $\left.\mathrm{Cl}, 30.2 \%, 32.9 \%, \mathrm{I}^{2}: 99.6 \%\right)$

\section{Meta-regression finding based on the mean of age and frequency of readmission:}

The studies' meta-regression was according to the association between frequency of readmission and the mean age of patients. There was no statistically significant linear trend in univariate meta-regression to explain effect size variation by mean of age of study with coefficient $=0.14(95 \% \mathrm{Cl}-2.17,2.46), \mathrm{P}=0.88$ (Fig. 3a).

\section{Meta-regression finding based on the male to female ratio of study and prevalence of readmission:}

The overall rate of readmissions based on the female to male ratio of the studies is showed in Figure. 4-b,the prevalence of readmission was lower in studies with higher male to female ratio. There was statistically significant linear trend in univariate meta-regression to explain effect size variation by male to female ratio of study with coefficient $=0.47(95 \% \mathrm{Cl} 0.03,1620.91), \mathrm{P}=0.03$. (Fig. 3b)

Meta-regression finding based on the mean of age and frequency of readmission:

The studies' meta-regression was according to the association between frequency of readmission and the publication year of included studies. There was no statistically significant linear trend in univariate metaregression to explain effect size variation by mean of age of study with coefficient $=0.14(95 \% \mathrm{Cl}-2.17$, 2.46), $P=0.88$ (Fig. 3c). 


\section{Publication Bias:}

Funnel plot in Figure (3d) shows no indication of publication bias. It is shows in funnel plot symmetrically. Circles' size shows the weight of studies (bigger circles shows more sample and smaller circles shows fewer sample).

\section{Discussion:}

It is well known that epilepsy surgery success rates range from $50-80 \% .(11,12)$ Hence, it is not surprising that patients might require readmission for additional monitoring or management of persistent seizures. There is a growing body of literature about 30-day readmission rates after neurosurgical interventions. Single neurosurgical centers have found overall readmission rates nearing $10 \%$, with most predictors of readmissions seen secondary to postoperative care complications(13-15). However, within this body of literature, those related to surgery in persons with epilepsy remain limited.The principle aim of this systematic review and meta-analysis was to determine the 30-day readmission rate in epilepsy surgery, which we found to be $10 \%$.all included studies were from USA and reported 30-day readmission using NSQIP database. Predictors of readmission has been reported to be male sex and initial admission via the emergency department(16), Medicare insurance ,lowest quartile of income, depression, hemispherectomy, postoperative complications, and small bed size hospital(17).in our study due to the lack of data in the included studies we could only analize some of the effective factors such as Payer, Mean Elixhauser index, Median annual household income, Lenghth of stay, Surgical complications, Medical complications, Hospital bed size and Median household income.our study showed no significant relationship between any of these factors and the readmission rate.the most common cause of readmission was seizure deppression has been reported to be a predictor of worse postsurgical seizure outcome, specifically, less likely to be seizure free $(18,19)$. the mean Elixhauser index was 8.05 and the mean length of stay was 2.95 days. Rumalla et al in their study evaluated the 90 day readmission too.they reported a $16.5 \% 90$ day readmission. They also indicated that Predictors of 90-d readmission, in addition to Medicare payer status and depression, included increasing number of comorbidities and medical complications(20). The most common medical complications likely resulted from mechanical ventilation and immobility or deep venous thrombosis (DVT). Therefore, additional attention is deserved for preventing these medical complications, ie, DVT prophylaxis, early ambulation, and incentive spirometry(21). Moghavem et al in their study stated that More evidence is required to identify the factors that may lead to improved outcomes, particularly modifiable factors. Some of our data suggest that many readmissions identified in our study are potentially preventable complications such as (postoperative infections, urinary tract infections,pneumonia, and pulmonary emboli). Thirty-day readmission metrics are now commonly studied as they can represent an indicator of quality of care and cost-effective management. Monetary incentives are distributed by healthcare regulations targeting quality improvement in inpatient management that is also cost-saving. Our results suggest that, particularly in Medicare recipients who tend to have more comorbid conditions, careful postoperative discharge planning that involves allied health services after neurosurgery and follow-up soon after discharge to minimize seizures or any signs of post-op complications may be important to enhance outcomes and reduce the risk of readmission and its associated consequences in epilepsy. 


\section{Limitation:}

First , It is possible that additional causes of readmission listed in secondary diagnosis fields were missed. Since second,the follow-up time was limited in some of the included studies. Our study may also be understating the risk of readmission for epilepsy surgery, as the data of included studies do not capture patients who die outside the hospital after their procedure or seek additional treatment in another state.

\section{Conclusion:}

Minimizing factors that contribute to readmission in various patient populations and procedures becomes important for patient care, resource utilization, and physician reimbursement.understanding about a readmission rate of $10 \%$ can be useful to health systems designing quality improvement efforts.Awareness of the reasons for readmission is important for patient counseling and surgical decision making. In epilepsy surgery, although our study did not show a significant difference but, readmissions might be reduced with optimization of pre-existing comorbidities.

\section{Abbrevations:}

Centers for Medicare \& Medicaid Services (CMS)

Agency for Healthcare Research Quality (AHRQ)

State Inpatient Database (SID)

length of stay (LOS)

National Surgical Quality Improvement Program(NSQIP)

deep venous thrombosis (DVT)

\section{Declarations}

\section{Ethics Approval and Consent to Participate:}

not applicable.

\section{Consent for publication:}

not applicable

\section{Competing Interests:}

Neither of the authors has any conflict of interest to disclose 


\section{Funding:}

not applicable

\section{Author contribution:}

ZM participated in Conception and design of the study, library searches and assembling relevant literature, critical review of the paper, supervising writing of the paper, Database management.FP participated in Data collection, library searches and assembling relevant literature, writing the paper, and critical review of the paper.MS participated in Data collection, library searches and assembling relevant literature, writing the paper, analysis of the data and critical review of the paper. All authors read and approved the final manuscript.

\section{Acknowledgement:}

not applicable

We confirm that we have read the Journal's position on issues involved in ethical publication and affirm that this report is consistent with those guidelines.

\section{References}

1. Vedantam A, Pan IW, Staggers KA, Lam SK. Thirty-day outcomes in pediatric epilepsy surgery. Child's Nervous System. 2018 Mar 1;34(3):487-94.

2. Amin BY, Tu TH, Schairer WW, Na L, Takemoto S, Berven S, Deviren V, Ames C, Chou D, Mummaneni PV. Pitfalls of calculating hospital readmission rates based on nonvalidated administrative data sets: Presented at the 2012 Spine Section Meeting. Journal of Neurosurgery: Spine. 2013 Feb 1;18(2):134-8.

3. Berry JG, Toomey SL, Zaslavsky AM, Jha AK, Nakamura MM, Klein DJ, Feng JY, Shulman S, Chiang VW, Kaplan W, Hall M. Pediatric readmission prevalence and variability across hospitals. Jama. 2013 Jan 23;309(4):372-80.

4. Chotai S, Guidry BS, Chan EW, Sborov KD, Gannon S, Shannon C, Bonfield CM, Wellons JC, Naftel RP. Unplanned readmission within 90 days after pediatric neurosurgery. Journal of Neurosurgery: Pediatrics. 2017 Dec 1;20(6):542-8.

5. Blank LJ, Crispo JA, Thibault DP, Davis KA, Litt B, Willis AW. Readmission after seizure discharge in a nationally representative sample. Neurology. 2019 Jan 29;92(5):e429-42.

6. Dasenbrock HH, Yan SC, Smith TR, Valdes PA, Gormley WB, Claus EB, Dunn IF. Readmission after craniotomy for tumor: a national surgical quality improvement program analysis. Neurosurgery. $2017 \mathrm{Apr}$ 1;80(4):551-62.

7. Dewan MC, White-Dzuro GA, Brinson PR, Thompson RC, Chambless LB. Perioperative seizure in patients with glioma is associated with longer hospitalization, higher readmission, and decreased overall survival. Journal of neurosurgery. 2016 Oct 1;125(4):1033-41. 
8. Kerezoudis P, McCutcheon B, Murphy ME, Rajjoub KR, Ubl D, Habermann EB, Worrell G, Bydon M, Van Gompel JJ. Thirty-day postoperative morbidity and mortality after temporal lobectomy for medically refractory epilepsy. Journal of neurosurgery. 2018 Apr 1;128(4):1158-64.

9. Ansari SF, Yan H, Zou J, Worth RM, Barbaro NM. Hospital length of stay and readmission rate for neurosurgical patients. Neurosurgery. 2018 Feb 1;82(2):173-81.

10. Sherrod BA, Johnston JM, Rocque BG. Risk factors for unplanned readmission within 30 days after pediatric neurosurgery: a nationwide analysis of 9799 procedures from the American College of Surgeons National Surgical Quality Improvement Program. Journal of Neurosurgery: Pediatrics. 2016 Sep 1;18(3):350-62.

11. Kim Y, Gani F, Lucas DJ, et al. Early versus late readmission after surgery among patients with employerprovided health insurance. Ann Surg. 2015;262(3):502-511.

12. Koubeissi MZ, Puwanant A, Jehi L, Alshekhlee A. In-hospital complications of epilepsy surgery: a six-year nationwide experience. Br J Neurosurg. 2009;23(5):524-529.

13. Shah MN, Stoev IT, Sanford DE, Gao F, Santiago P, Jaques DP, et al. Are readmission rates on a neurosurgical service indicators of quality of care? J Neurosurg. 2013;119(4):1043-9.

14. Marcus LP, McCutcheon BA, Noorbakhsh A, Parina RP, Gonda DD, Chen C, et al. Incidence and predictors of 30-day readmission for patients discharged home after craniotomy for malignant supratentorial tumors in California (1995-2010). J Neurosurg. 2014;120(5):1201-11.

15. Buchanan CC, Hernandez EA, Anderson JM, Dye JA, Leung M, Buxey F, et al. Analysis of 30-day readmissions among neurosurgical patients: surgical complication avoidance.

16. Moghavem N, Morrison D, Ratliff JK, Hernandez-Boussard T. Cranial neurosurgical 30-day readmissions by clinical indication.J Neurosurg. 2015;123(1):189-97.

17. Buchanan CC, Hernandez EA, Anderson JM, Dye JA, Leung M,Buxey F, et al. Analysis of 30-day readmissions among neurosurgical patients: surgical complication avoidance as key to quality improvement. J Neurosurg. 2014;121(1):170-5.

18. Wilson SJ, Kincade P, Saling MM, Bladin PF. Patient readmission and support utilization following anterior temporal lobectomy. Seizure. 1999;8(1):20-25.

19. Kanner AM BR, Smith MC, Balabanov AJ, Frey M. Does a life-time history of depression predict a worse post-surgical seizure outcome following temporal lobectomy? Ann Neurol. 2006;60(Suppl 3):S19.

20. Rumalla K, Smith KA, Arnold PM, Schwartz TH. Readmission following surgical resection for intractable epilepsy: nationwide rates, causes, predictors, and outcomes. Operative Neurosurgery. 2019 Mar $1 ; 16(3): 374-82$.

21. Pavon JM, Adam SS, Razouki ZA, et al. Effectiveness of intermittent pneumatic compression devices for venous thromboembolism prophylaxis in High-Risk surgical patients: A systematic review. J Arthroplasty. 2016;31(2):524-532.

22. Vedantam A, Pan IW, Staggers KA, Lam SK. Thirty-day outcomes in pediatric epilepsy surgery. Child's Nervous System. 2018 Mar 1;34(3):487-94.

23. Kwon CS, Agarwal P, Subramaniam V, Dhamoon M, Mazumdar M, Yeshokumar A, Panov F, Ghatan S, Jetté N. Readmission after neurosurgical intervention in epilepsy: A nationwide cohort analysis. 
Epilepsia. 2020 Jan;61(1):61-9.

24. Kalanithi PS, Arrigo RT, Tran P, Gephart MH, Shuer L, Fisher R, Boakye M. Rehospitalization and emergency department use rates before and after vagus nerve stimulation for epilepsy: use of state databases to provide longitudinal data across multiple clinical settings. Neuromodulation: Technology at the Neural Interface. 2014 Jan;17(1):60-5.

25. Vawter-Lee M, Lutley A, Lake SW, Fledderjohn S, King A, Horn PS, Wesselkamper KR. Pediatric Epilepsy Readmissions: The Who, When, and Why. Pediatric neurology. 2019 Apr 1;93:11-6.

26. Subramaniam V, Kwon CS, Agarwal P, Dhamoon M, Mazumdar M, Jette N. Hospital Readmissions After an Epilepsy Related Surgical Intervention in the Nationwide Readmissions Database (P5. 5-024).

\section{Figures}

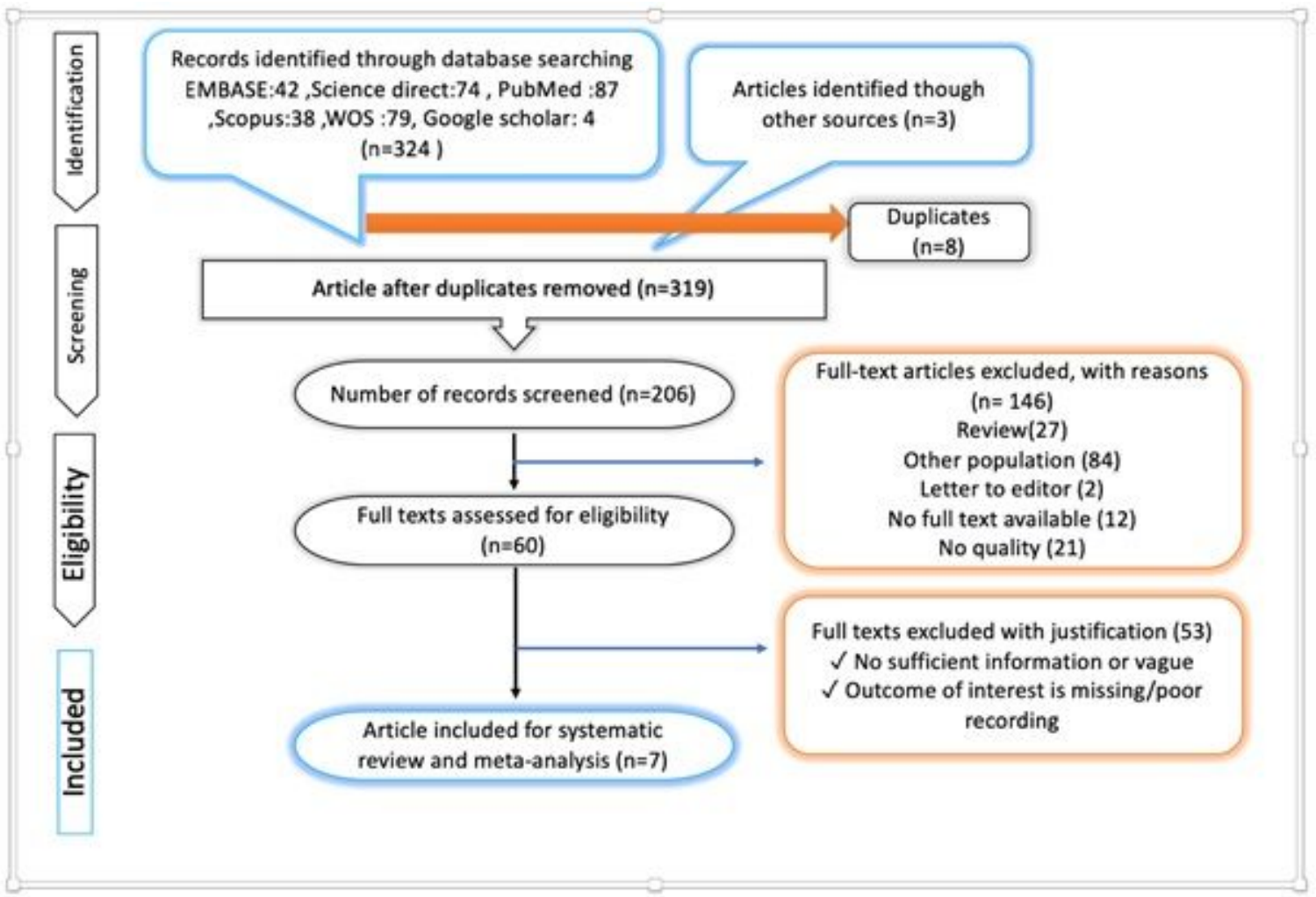

\section{Figure 1}

PRISMA flow diagram 


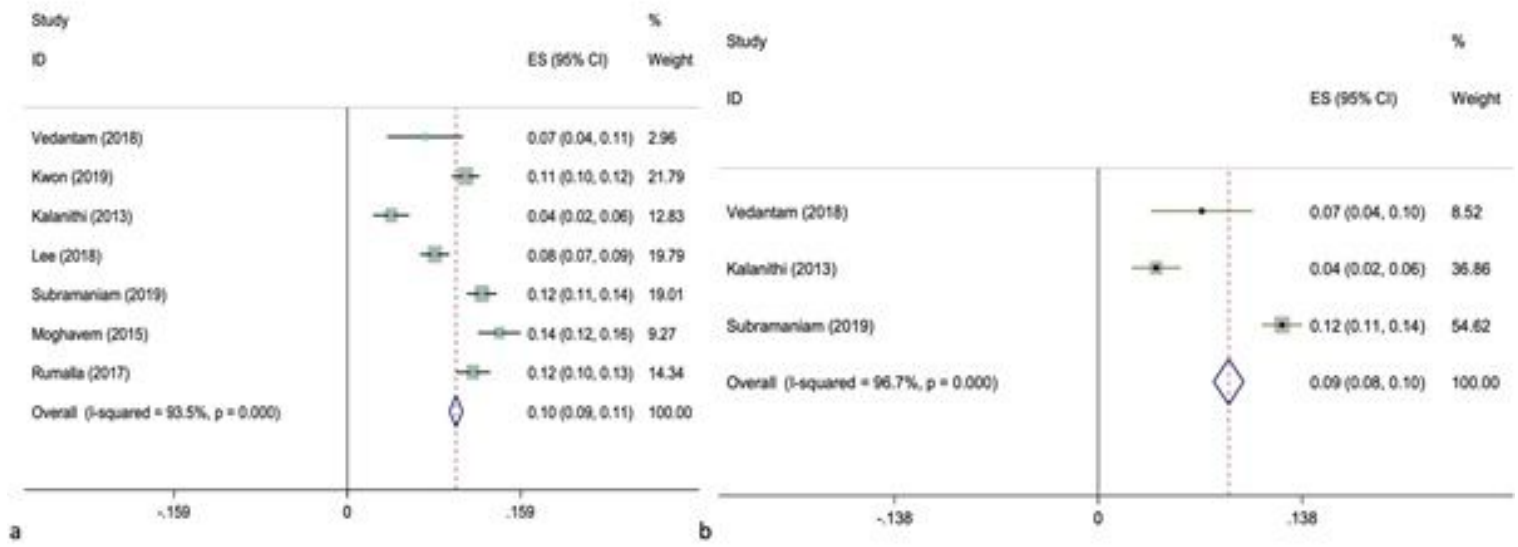

\section{Figure 2}

Meta-analysis of the readmission and re-operation prevalence
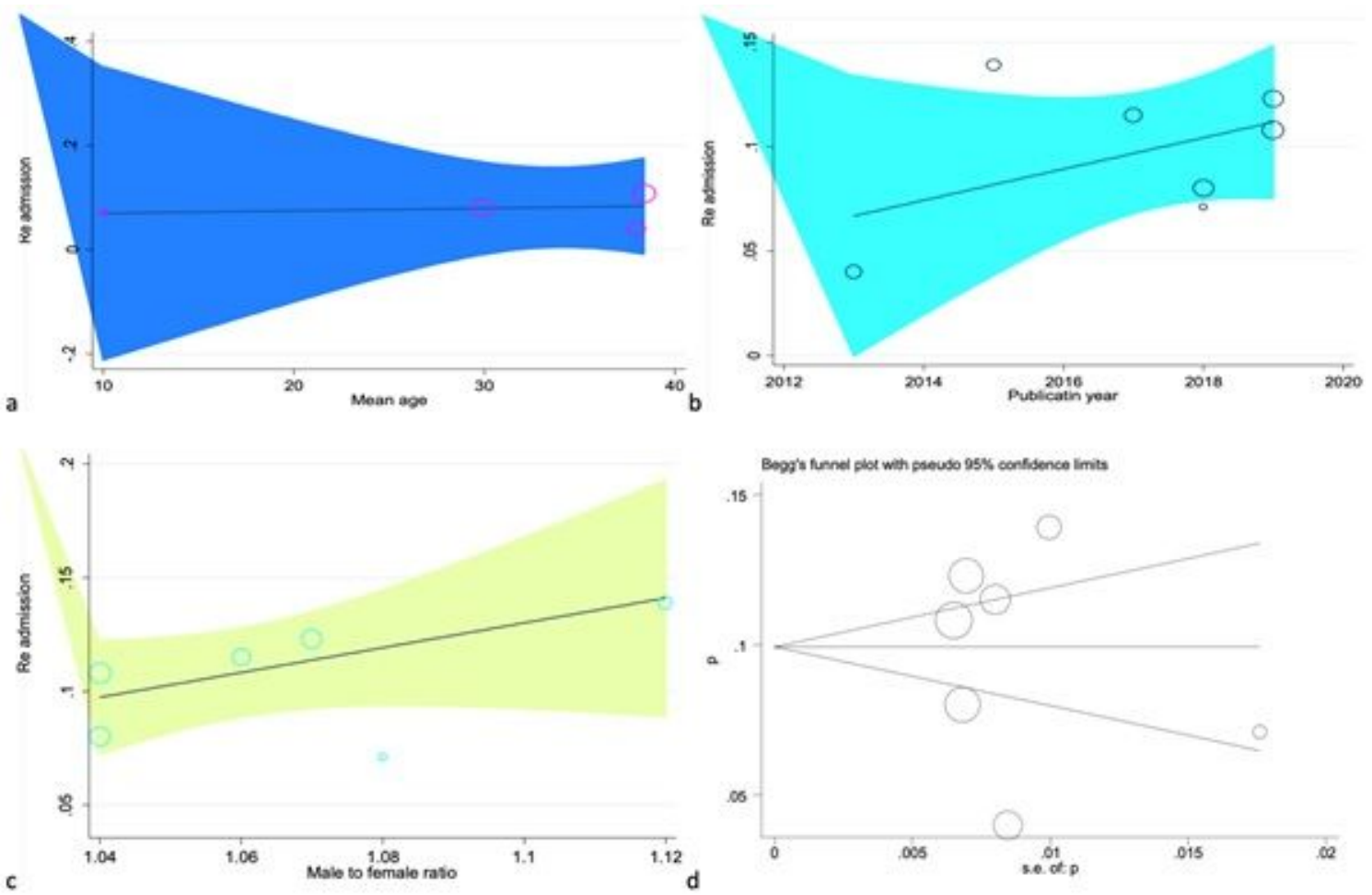

Figure 3

Meta-regression finding based on the mean of age(a),publication year(b),male to female ratio(c) and frequency of readmission. Begg's funnel plot for publication bias (d) 